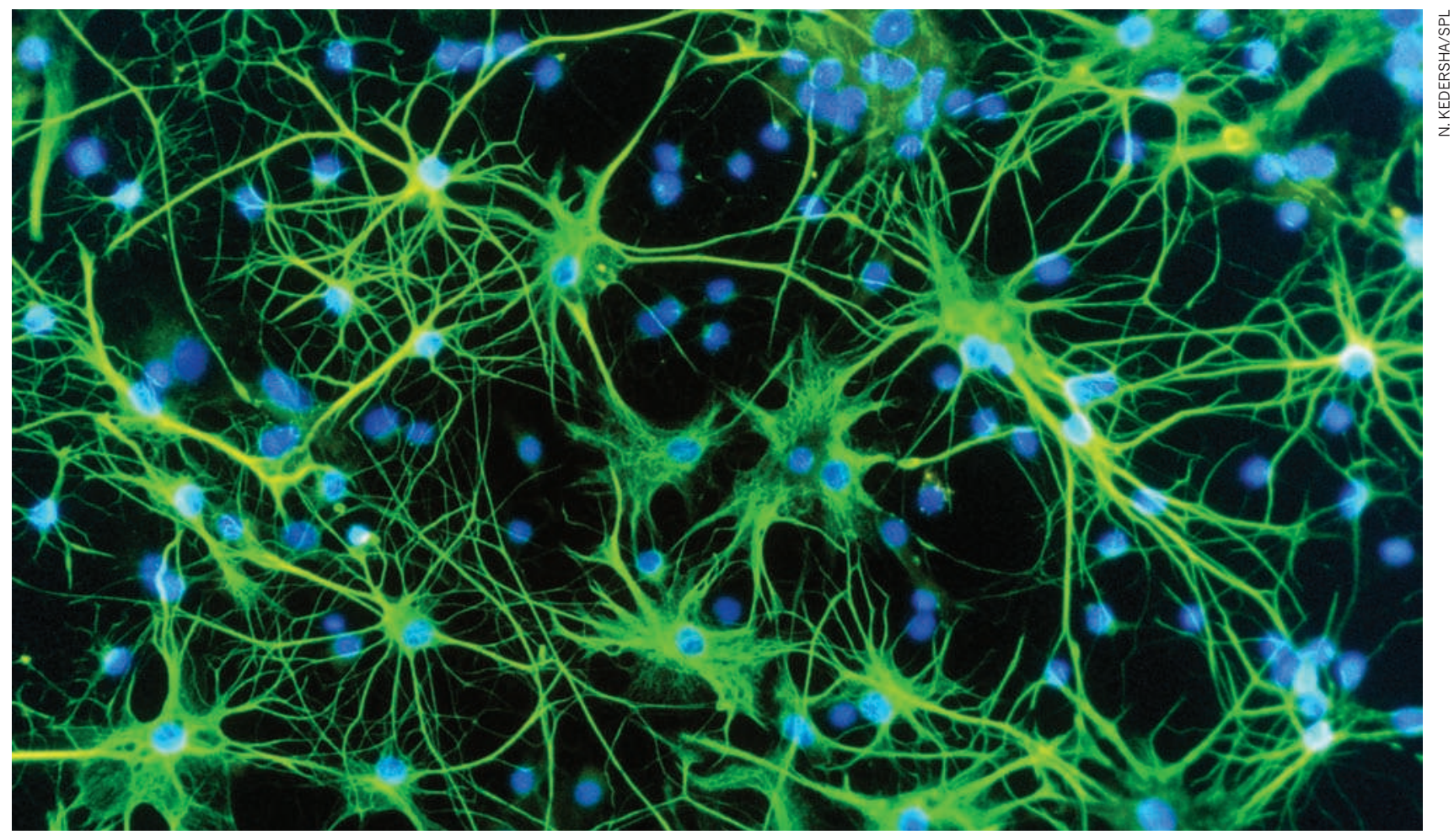

Astrocytes (stained green) form rich networks in the brain, which may increase the complexity of neural processing.

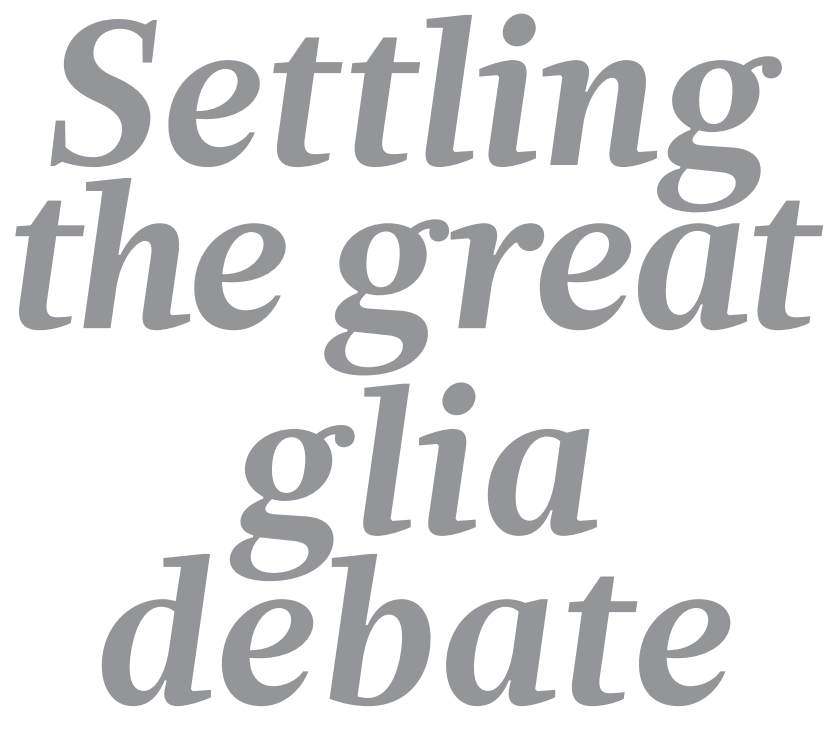

Do the billions

of non-neuronal cells in the brain send messages of their own?
$\mathrm{H}$ alfway through a satellite meeting at the Federation of European Neurosciences conference in Amsterdam in July, researcher Ken McCarthy takes the stage to give his presentation. He sports a black shirt and jeans, and his strong cheekbones, shock of white hair and tanned skin give him the look of a film star. But he doesn't have the confidence to match. "I find this a little bit daunting," he says, as he organizes his slides.

McCarthy, a geneticist at the University of North Carolina School of Medicine in Chapel Hill, is about to fan the flames of a debate about whether glia, the largest contingent of non-neuronal cells in the brain, are important in transmitting electrical messages. For many years, neurons were thought to be alone in executing this task, and glia were consigned to a supporting role - regulating a neuron's environment, helping it to grow, and even providing physical scaffolding (glia is Greek for 'glue').

In the past couple of decades, however, this picture has been changing. Some glia, known as astrocytes, have thousands of bushy tendrils that nestle close to the active junctions between neurons - the synapses (see 'Neural threesome'). Here they seem to listen in on neuronal activity and, in turn, to influence it. Studies show that chemical transmitters released by neurons cause an increase in the levels of calcium inside astrocytes, spurring them to release transmitters of their own. These can enhance or mute the signalling between neurons, or influence the strength of their connections over time. Moreover, astrocytes activated at one synapse might communicate with other synapses and astrocytes with which they make contact.

The consequences of this 'gliotransmission' could be profound. The human brain contains roughly equal numbers of glia and neurons (about 85 billion of each), and any given astrocyte can make as many as 30,000 connections with cells around it. If glia are involved in signalling, processing in the brain turns out to be an order of magnitude more complex than previously expected, says 
Andrea Volterra, who studies astrocytes at the University of Lausanne in Switzerland. Neuroscientists, who have long focused on the neuron, he says, would "have to revise everything". In the past year or so, several papers have highlighted the urgency of this revision.

But the research that McCarthy is about to discuss could put a stop to the enthusiasm. "I'm presenting work from genetic studies that fly in the face of gliotransmission," he begins. Most studies so far have investigated astrocytes cultured in dishes, and bombarded them with calcium to elicit an effect. It has long been suggested, however, that these methods aren't specific enough to astrocytes, and might be affecting neurons as well. What is needed is a way to target astrocytes alone. So McCarthy has developed genetically engineered mice in which astrocytes can't signal normally. The mutations seem to have no effect on neuronal transmission in the brain.

His group's finding could come as a relief to some. Given the enormous neural complexity that gliotransmission would imply, "people don't want astrocytes to be involved", says Phil Haydon, a neuroscientist who studies glia at Tufts University in Boston, Massachusetts. But many, including most at the Amsterdam meeting, have built their careers on gliotransmission. In addition to their effects on the day-to-day functioning of the central nervous system, glia have opened new avenues of research into sleep, as well as psychiatric and neurological disease. Now, researchers are being forced to prove McCarthy wrong, or re-evaluate the fundamental precepts upon which the field was built. David Attwell, a neuroscientist at University College London, says that emotions in the community are running high. "If someone comes along and says that everything you've done is wrong, it's like you've wasted your life," he says. "It's become quite a polarized field, just in the last year or two."

\section{The bomb}

Gliotransmission has had plenty of support recently. In January this year, a group led by Dmitri Rusakov at University College London and Stéphane Oliet at the University of Bordeaux in France published results ${ }^{1}$ suggesting that the chemical $\mathrm{D}$-serine, released from astrocytes, activates a particular receptor - the NMDAR, or N-methyl-D-aspartate receptor - on the surface of neurons, influencing their behaviour. Communication through NMDARs is thought to be important in learning and memory, because it can help to enhance chatter between synapses and help memories to form.

And in September, a team led by Justin Lee at the Korea Institute of Science and Technology in Seoul found evidence ${ }^{2}$ that astrocytes in the cerebellum release the neurotransmitter GABA ( $\gamma$-aminobutyric acid). However, unlike the work of most groups, which suggests that astrocytes release chemicals packaged within tiny bubbles called vesicles, Lee's experiments imply that the cells are transmitting the chemicals directly through an ion channel in their membranes. When the researchers blocked the channel, called Bestrophin-1, GABA levels went down, suggesting that glia release GABA through this outlet.

But there have been concerns about experimental techniques. In these experiments, and many before them, investigators have tried to induce astrocyte signalling in petri dishes by pipetting calcium into individual cells and watching what happens. This approach is "like an atom bomb" going off in the cell, says McCarthy. Under natural conditions, the level of calcium would increase much more slowly, and McCarthy is concerned that pumping a cell full of calcium could make it behave strangely perhaps forcing it to produce transmitters, or even preventing

it from releasing anything. McCarthy hoped to unpick the role of glia in the brain using his genetically engineered mice. "We thought this would definitively show gliotransmission," he says.

Instead, it totally crushed expectations. The team, led by McCarthy's postdoc, Cendra Agulhon, studied two mouse lines: one in which calcium signalling in astrocytes had been given a boost, and another in which it had been completely obliterated. But neither change made any difference to how nearby neurons were going about their business. McCarthy and his group were forced to conclude that astrocytes couldn't possibly be releasing chemicals to signal to neurons. They published their results in Science in March this year ${ }^{3}$.

At the meeting in Amsterdam, McCarthy discusses his group's ongoing search for a behavioural effect that they could attribute to their wonky astrocytes. Again, no dice. "I would love to be able to show you that, but I can't," McCarthy says, amid murmurs of disbelief from the audience.

But some researchers have problems with his model. "The way he does it bothers me," says Richard Robitaille, a glia biologist at the University of Montreal in Canada. Measuring the effect of astrocytes will require more subtle experimental approaches, he says. Haydon agrees that it would be better to use more sensitive methods. He pictures astrocyte responses to stimuli as a narrow bell curve: if you blast them with something, they do nothing; if you don't stimulate them at all, they do nothing. But 'talk' to them using a low level of physiologically relevant stimulation and they should talk back. In McCarthy's mice, says Haydon, taking out the activity of all astrocytes probably changes so much - during brain development, and throughout life - that it is impossible to tell what the normal job of glia might be and how the brain compensates for their absence. The mice are thus subject to the same 'atom bomb' criticism levelled at cell-culture studies.

Part of the problem could be cultural. "The entire field has been trained in neurocentric labs, and everybody has so far believed that astrocytes work like neurons," says Maiken Nedergaard, a glial biologist at the University of Rochester in New York. "But astrocytes function totally differently. They use a different language. They use a different way of getting input and output." They may also work on a totally different timescale from neurons, says Rusakov. Their responses, he says, can be three orders of magnitude slower. As a consequence, common techniques for measuring neuronal responses won't work on astrocytes. Methods for imaging calcium in cells aren't good at measuring slow fluctuations or increases in the outer reaches of astrocytes, partly because calcium dyes simply don't penetrate there.

At his lab at the University of California, Los Angeles, Baljit Khakh has been developing a technique that can detect calcium in the previously inaccessible branches of astrocytes ${ }^{4}$. His team took an existing protein that is known to sense calcium, and modified it so that it could be targeted to cell membranes, where researchers suspect much of the calcium signalling might be going on.

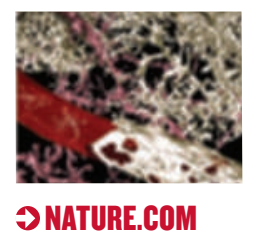

For more on glia research, visit: go.nature.com/7u635r
His work shows that a calcium increase in the main body of the astrocyte didn't necessarily cause a corresponding rise in calcium at the borders. If glia are indeed releasing transmitters, methods such as this should allow researchers to examine exactly where they are releasing them from.

Robitaille aims to find out whether astrocytes can detect very low levels of synaptic transmission - a neuron passing just 


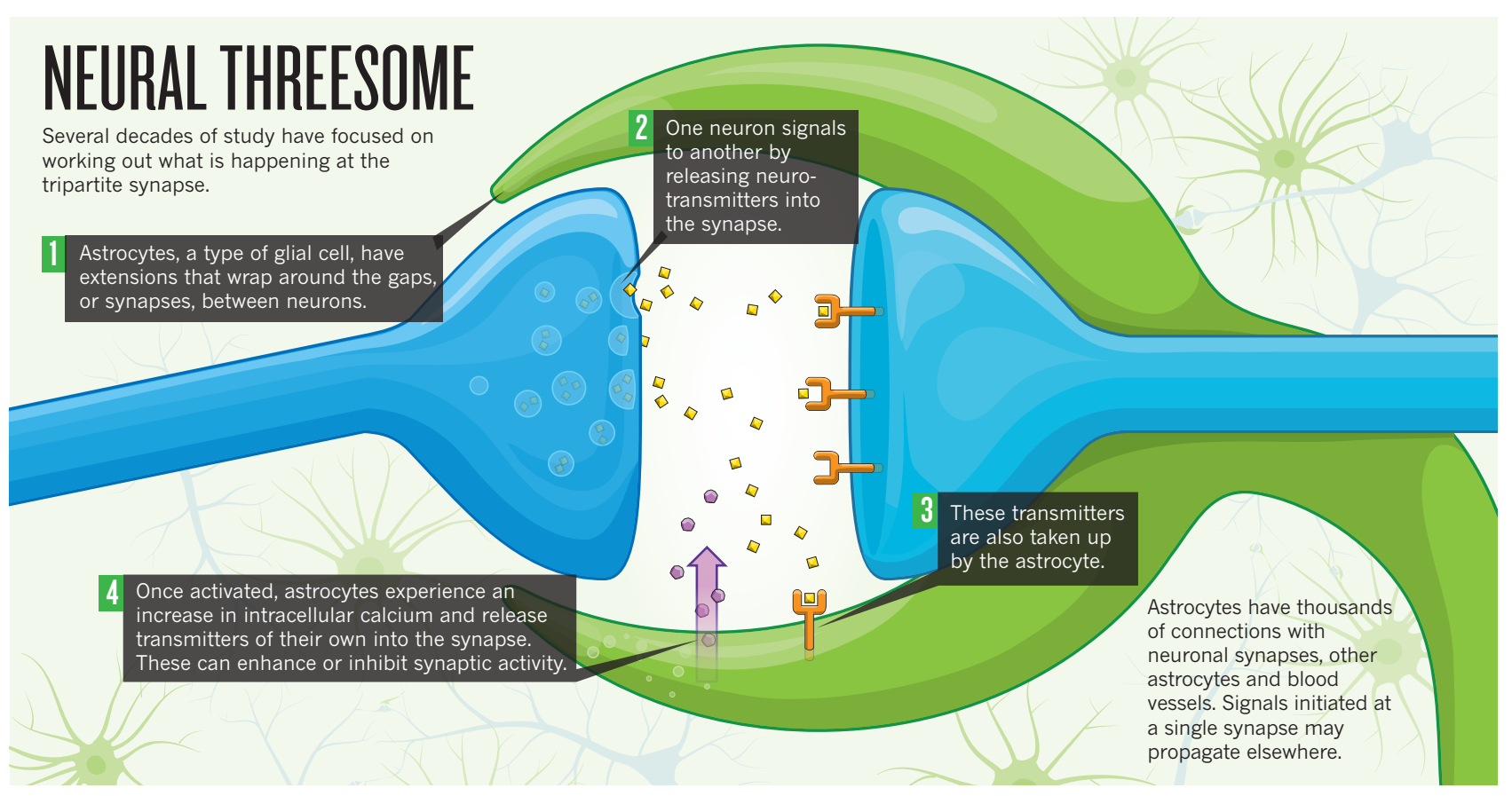

one electrical signal, or action potential, to the next. He and a colleague, Aude Panatier, used a high-speed imaging technique called line-scan imaging to measure calcium in astrocyte branches, and activated a small number of neurons using a very weak electrical pulse. Their results, not yet published, suggest that astrocytes can indeed pick up these low levels of activity and, what's more, they can regulate transmission themselves by releasing chemicals such as the energy-transfer molecules adenosine and ATP. Robitaille and his team have come down in favour of gliotransmission. "Based on our data, we have to sit on one side of the fence," he says.

Studies in different areas of the brain could also be contributing to the dramatically varying results. "Our knowledge about astrocytes is so poor that people tend to generalize findings in different circuits, different brain areas," says Volterra. "That also should be looked at." Even the time of day at which brains are prepared for experiments could be affecting measurements, says Haydon, because certain chemicals wax and wane over time. "I have a student go in and cut slices every four hours," he says at the conference, to a ripple of sympathetic chuckling from the audience.

\section{The killer experiment}

Methods aside, there doesn't seem to be a consensus as to what experiment would resolve this dilemma once and for all. "There's no clear simple experiment, otherwise I'd do it," says Attwell. "So would most of the others."

Most researchers in the field say that standardizing their methods and the ways in which they interpret their results could help. But the bigger personalities don't shy away from making ambitious statements. "Lots of neuron-centric people would look at our Science paper and say, 'That's the killer experiment,"' says McCarthy after his talk.

Later the same day, Haydon is gearing up to present some new results from his experiments on transgenic mice. When does he think the argument will be resolved? "Today," he says, "at 4.15."

Haydon's team works with mice with a different deficit to those that McCarthy is studying, although the two men - despite obvious differences of opinion - do share mouse resources and expertise. Haydon's mice have higher than normal levels of an enzyme called $\mathrm{IP}_{3}$ phosphatase, which acts to prevent astrocytes releasing calcium. But the impairment in the mice that Haydon uses is more precise than that in McCarthy's animals, in terms of both timing and location. The calcium-blocking enzyme is expressed only after the mice are weaned, and only in a brain region called the hippocampus.

Haydon shows the assembled audience evidence that calcium signalling decreases in mice that have had their astrocytes disabled in this way, and that synaptic transmission in the hippocampus is also affected - implying that astrocytes help synaptic transmission to take place, under the right conditions. The work suggests that with heavy stimulation, synaptic transmission isn't changed; likewise, with a very weak stimulus, the astrocytes make no contribution. But there is a 'window of opportunity', at a medium level of stimulation, in which the effects are visible.

This is music to the ears of many glia enthusiasts. Even McCarthy is pleased to hear about positive results. "I would love to see gliotransmission," he says. But the evidence both for and against it still needs to be verified. With different groups approaching the question in different ways, and with the bestpractice methods and techniques yet to be standardized and agreed on, it will take time before researchers can replicate each other's results and bring some clarity to the field.

McCarthy for one will keep searching for a behavioural effect in his mice with altered astrocytes, but is wary of letting what he wants to see taint his results. "If you get a vested interest in the answer, then you're in trouble," he says. Nonetheless, "If we do see it, we're going to be shouting it from the rooftops." SEE INSIGHT, P.213

Kerri Smith is editor of the Nature Podcast, writing for Nature from London.

1. Henneberger, C., Papouin, T., Oliet, S. H. R. \& Rusakov, D. A. Nature 463, 232-236 (2010).

2. Lee, S. et al. Science doi:10.1126/science.1184334 (2010).

3. Agulhon, C., Fiacco, T. A. \& McCarthy, K. D. Science 327, 1250-1254 (2010).

4. Shigetomi, E., Kracun, S., Sofroniew, M. V. \& Khakh, B. S. Nature Neurosci. 13, 759-766 (2010). 